\title{
Spin models on random lattices
}

\author{
Wolfhard Janke ${ }^{\mathrm{a}, *}$, Desmond A. Johnston ${ }^{\mathrm{b}}$, Ramon Villanova ${ }^{\mathrm{c}}$ \\ ${ }^{a}$ Institut für Theoretische Physik, Universität Leipzig, Augustusplatz 10/11, 04109 Leipzig, Germany \\ ${ }^{\mathrm{b}}$ Department of Mathematics, Heriot-Watt University, Edinburgh, EH144AS, Scotland, UK

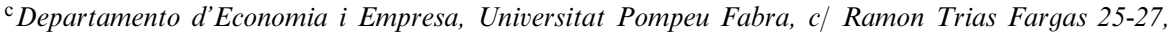 \\ 08005 Barcelona, Spain
}

\begin{abstract}
Under certain conditions phase transitions in systems with quenched disorder are expected to exhibit a different behaviour than in the corresponding pure system. Here we discuss a series of Monte Carlo studies of a special type of such disordered systems, namely spin models defined on quenched, random lattices exhibiting geometrical disorder in the connectivity of the lattice sites. In two dimensions we present results for the $q$-state Potts model on random tri-valent $\left(\Phi^{3}\right)$ planar graphs, which appear quite naturally in the dynamically triangulated random surface (DTRS) approach to quantum gravity, as well as on Poissonian random lattices of Voronoi/Delaunay type. Both cases, $q \leqslant 4$ and $>4$, are discussed which, in the pure model without disorder, give rise to second- and first-order phase transitions, respectively. In three dimensions results for the Ising model on Poissonian random lattices are briefly described. We conclude with a comparison of the two types of connectivity disorder with the more standard case of bond disorder, and a discussion of the distinguishing differences. (c) 2000 Elsevier Science B.V. All rights reserved.
\end{abstract}

Keywords: Connectivity disorder; Random lattices; Gravity graphs; Potts model; Phase transitions; Monte Carlo simulations

\section{Introduction}

In the past decade much work has been devoted to a deeper understanding of quenched, disordered systems. Despite all these efforts there is still no little controversy regarding the critical behaviour of such systems, even in seemingly simple two-dimensional (2D) systems with quenched bond disorder (for a review see, [1]). It has been known for some time that the first-order phase transition displayed by

\footnotetext{
${ }^{*}$ Corresponding author. Fax: +49-341-97-32-747.

E-mail addresses: janke@itp.uni-leipzig.de (W. Janke), des@ma.hw.ac.uk (D.A. Johnston), villanov@upf.es (R. Villanova)
} 
$q>4$-state Potts models on regular lattices is softened by the introduction of the quenched bond disorder to a continuous transition [2-5], though the universality class of this transition and its dependence on the strength and nature of the bond disorder are still not completely clear [6-12]. Models which already display a continuous transition in the pure case appear to have their critical exponents altered by the bond disorder [13-26] provided that the critical exponent $\alpha_{\text {pure }}$ of the specific heat is positive. In addition, a qualitatively new phenomenon in the form of multi-fractal scaling of local correlators has also been predicted [27] and measured [28] when quenched bond disorder is present. It should be remarked that all of the theoretical results in the bond disordered case are perturbative in nature, which is in large measure the source of the controversy surrounding the various predictions for critical exponents, since the domain of validity is unclear.

Another type of disorder that might be imposed is quenched connectivity disorder. Here all coupling constants are the same but the number of interacting nearest-neighbour spins varies from site to site of a random lattice or graph. In the next section we describe two different random lattice constructions, Poissonian Voronoi/Delaunay random lattices and tri-valent $\left(\Phi^{3}\right)$ gravity graphs which, as we argue in the following sections, seem to induce quite different effects on the phase transition in Ising and Potts spin systems.

\section{Random lattices and graphs}

\subsection{Voronoi/Delaunay random lattices}

The first type of connectivity disorder we have investigated is generated by Poissonian Voronoi/Delaunay random lattices which can be constructed in arbitrary dimensions as follows [29,30]:

- Draw $N$ sites $x_{i}$ at random in a unit volume (square in $2 \mathrm{D}$, cube in $3 \mathrm{D}, \ldots$ ).

- Associate with each site $x_{i}$ a Voronoi cell $c_{i} \equiv\left\{x \mid d\left(x, x_{i}\right) \leqslant d\left(x, x_{j}\right)\right.$ f.a. $\left.j \neq i\right\}$ (polygons in 2D, polyhedra in $3 \mathrm{D}, \ldots$ ), which consists of all points $x$ whose Euclidean distance $d\left(x, x_{i}\right)$ to the center site $x_{i}$ is smaller than to any other site.

- Construct the dual Delaunay lattice by linking the centre sites of Voronoi cells which share a common face.

Using this construction the connectivity of the lattice, i.e., the local coordination number $q_{i}$, varies from site to site according to the probability density $P\left(q_{i}\right)$ shown in Fig. 1 for the $2 \mathrm{D}$ case.

\subsection{Planar $\Phi^{3}$ gravity graphs}

The second type of connectivity disorder is motivated by numerical simulations of $2 \mathrm{D}$ quantum gravity where one studies dynamically triangulated random surfaces (DTRS) interacting with matter fields. The fluctuating geometry is supposed to realize the 


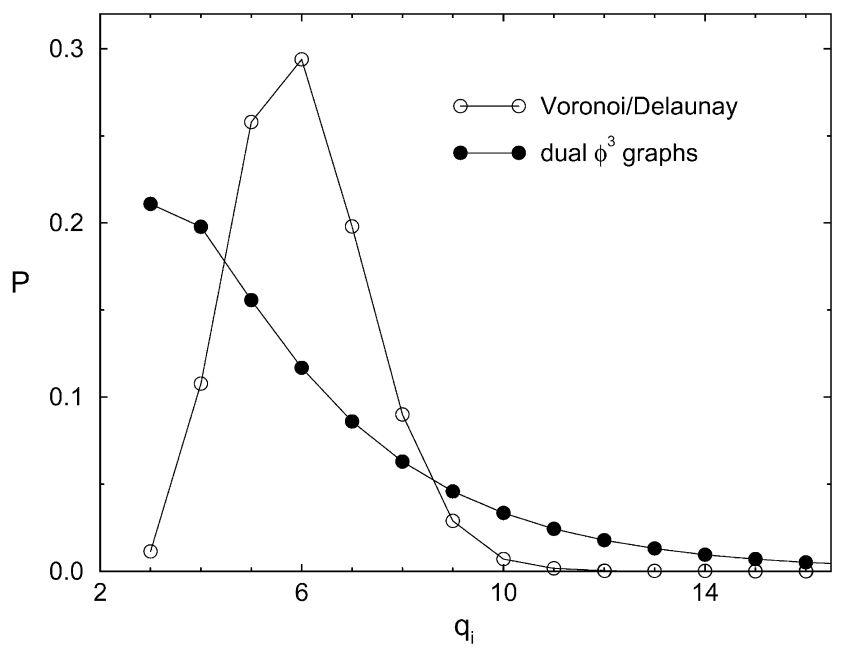

Fig. 1. Probability density of local coordination numbers for 2D Poissonian Voronoi/Delaunay random lattices and gravity triangulations dual to planar $\Phi^{3}$ graphs.

functional integration over the degrees of freedom of the metric tensor and can be generated in a Monte Carlo (MC) procedure by link-flip moves applied to tri-valent $\left(\Phi^{3}\right)$ planar graphs [31-35]. In the simplest case matter fields are approximated by spins on the vertices of these dynamical graphs. In the quantum gravity and string theory context one is therefore interested in annealed rather than quenched connectivity disorder, in which the graphs and spins are interacting on the same time scale, providing a discrete analogue of the back reaction in continuum theories of gravity. In the annealed case the relation between the bare $(\Delta)$ and dressed $(\tilde{\Delta})$ conformal weights is given by the KPZ relation [36-38]

$$
\tilde{\Delta}=\frac{\sqrt{1-c+24 \Delta}-\sqrt{1-c}}{\sqrt{25-c}-\sqrt{1-c}},
$$

where $c$ is the central charge of the matter fields. This allows us to calculate the standard critical exponents $\alpha=\left(1-2 \Delta_{\varepsilon}\right) /\left(1-\Delta_{\varepsilon}\right)$ and $\beta=\Delta_{\sigma} /\left(1-\Delta_{\varepsilon}\right)$ using the conformal weights $\Delta_{\varepsilon}$ and $\Delta_{\sigma}$ of the energy and spin density operators in both the dressed and undressed cases.

Here we are interested in a quenched graph ensemble, and for this purpose pure gravity graphs (i.e., graphs not affected by matter fields) can be much more easily generated with the Tutte algorithm [39-41] than by performing an actual DTRS MC simulation. The resulting probability density $P\left(q_{i}\right)$ looks completely different to Voronoi/Delaunay lattices, see Fig. 1 for a comparison. In the quenched case the dressed weights should simply follow $[42,43]$ by taking $c=0$ in the KPZ relation (1),

$$
\tilde{\Delta}_{\text {quenched }}=(\sqrt{1+24 \Delta}-1) / 4 \text {, }
$$

which now in general gives non-rational weights and exponents. 


\section{Simulations and data analysis}

In both the $\Phi^{3}$ and Voronoi simulations we used the standard definition of the $q$-state Potts model partition function and energy,

$$
Z_{\mathrm{Potts}}=\sum_{\left\{\sigma_{i}\right\}} \mathrm{e}^{-\beta E}, \quad E=-\sum_{\langle i j\rangle} \delta_{\sigma_{i} \sigma_{j}}, \quad \sigma_{i}=1, \ldots, q,
$$

where $\beta=J / k_{B} T$ is the inverse temperature in natural units, $\delta$ is the Kronecker symbol, and $\langle i j\rangle$ denotes the nearest-neighbour bonds of random lattices with $N$ sites labeled by the subscript $i$. We always used the Wolff single-cluster MC algorithm $[44,45]$ to update the spins $\sigma_{i}$. For each $N$ we generated an ensemble of independent random lattices, equilibrated the spin configurations and then performed a large number of measurement sweeps for each lattice realization. Between measurements we performed $n_{\text {flip }}$ cluster flips, with the average number of updated spins per flip being proportional to the average cluster size $\langle|C|\rangle$. The primary runs were carried out close to the transition point and time series of the energy $E$ and the magnetisation $M=\left(q \max \left\{n_{i}\right\}-N\right) /(q-1)$ recorded for each graph. The $n_{i} \leqslant N$ denote the numbers of spins of "orientation" $i=1, \ldots, q$ in one lattice configuration. In what follows the per-site quantities are denoted by $e=E / N$ and $m=M / N$, the thermal averages on each individual lattice by $\langle\ldots\rangle$ and the quenched average over the ensemble of independent random lattices by $[\ldots]_{\mathrm{av}}$.

From the time series of $e$ and $m$ it is straightforward to compute in the finite-size scaling (FSS) region various quantities at nearby values of $\beta$ by standard reweighting [46] techniques. Some care must be taken with the reweighting range in the presence of quenched averaging, but we confirmed that direct measurements of both the susceptibility and specific heat from fluctuations and numerical derivatives were in accordance with the values deduced from reweighting in several representative cases. A typical example for $\Phi^{3}$ graphs with $N=2000$ sites and $q=10$ is shown in Fig. 2.

To estimate the statistical (thermal) errors for each of the realizations, the time-series data was split into bins, which were jack-knifed [47,48] to decrease the bias in the analysis of reweighted data. The final values are averages over the realizations and the error bars are computed from the fluctuations among the realizations. Note that these errors contain both the average thermal error for a given realization and the theoretical variance for infinitely accurate thermal averages which is caused by the variation over the random lattices.

From the time series of the energy measurements we compute by reweighting the average energy $u=[\langle e\rangle]_{\mathrm{av}}$, the specific heat $C=\beta^{2} N\left[\left\langle\mathrm{e}^{2}\right\rangle-\langle e\rangle^{2}\right]_{\mathrm{av}}$, and the energetic fourth-order cumulant $V^{(1)}=\left[1-\left\langle\mathrm{e}^{4}\right\rangle / 3\left\langle\mathrm{e}^{2}\right\rangle^{2}\right]_{\mathrm{av}}$. Similarly, we derive from the magnetisation measurements the average magnetisation $[\langle|m|\rangle]_{\mathrm{av}}$, the susceptibility $\chi=\beta N\left[\left\langle m^{2}\right\rangle-\langle|m|\rangle^{2}\right]_{\mathrm{av}}$, and magnetic cumulants $U_{2 p}^{(1)}=\left[1-\left\langle m^{2 p}\right\rangle / 3\left\langle|m|^{p}\right\rangle^{2}\right]_{\mathrm{av}}$ with $p=1,2$, as well as the derivatives $\mathrm{d}[\langle|m|\rangle]_{\mathrm{av}} / \mathrm{d} \beta, \mathrm{d} \ln [\langle|m|\rangle]_{\mathrm{av}} / \mathrm{d} \beta$, and $\mathrm{d} \ln \left[\left\langle m^{2}\right\rangle\right]_{\mathrm{av}} / \mathrm{d} \beta$, which involve both the energy and magnetisation. 


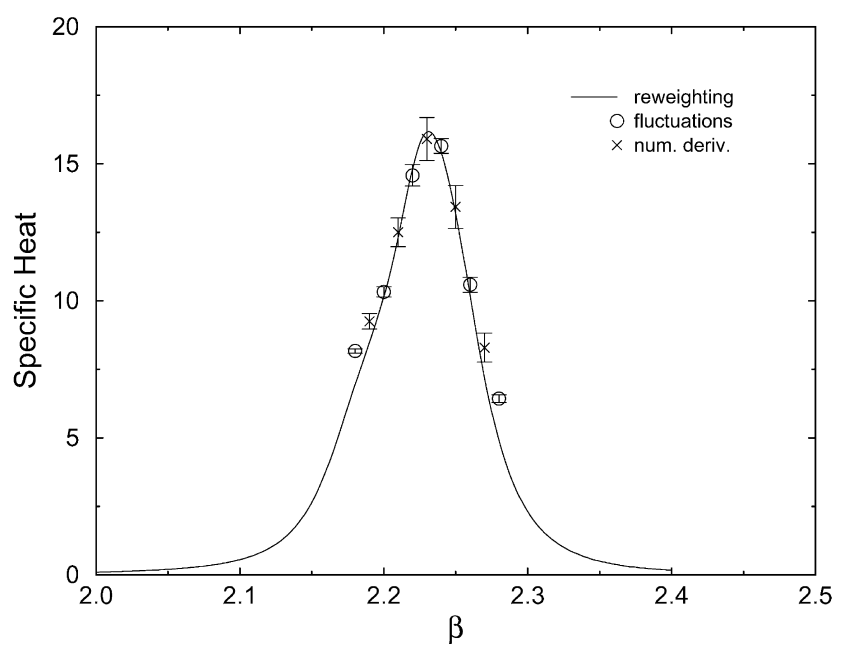

Fig. 2. The specific heat calculated from reweighting about $\beta_{0}=2.22$, fluctuations and numerical differentiation of the energy for $q=10$ and $N=2000 \Phi^{3}$-graphs (which is typical).

In the infinite-volume limit the various measured quantities exhibit singularities at the transition point. In finite systems the singularities are smeared out and scale in the critical region according to

$$
\begin{aligned}
& C=C_{\mathrm{reg}}+N^{\alpha / v D} f_{C}(x)[1+\cdots], \\
& \chi=N^{\gamma / v D} f_{\chi}(x)[1+\cdots], \\
& {[\langle|m|\rangle]_{\mathrm{av}}=N^{-\beta / v D} f_{m}(x)[1+\cdots],} \\
& \frac{\mathrm{d}[\langle|m|\rangle]_{\mathrm{av}}}{\mathrm{d} \beta}=N^{(1-\beta) / v D} f_{m^{\prime}}(x)[1+\cdots], \\
& \frac{\mathrm{d} \ln \left[\left\langle|m|^{p}\right\rangle\right]_{\mathrm{av}}}{\mathrm{d} \beta}=N^{1 / v D} f_{p}(x)[1+\cdots], \\
& \frac{\mathrm{d} U_{p}^{(1)}}{\mathrm{d} \beta}=N^{1 / v D} f_{U_{2 p}}(x)[1+\cdots],
\end{aligned}
$$

where $C_{\text {reg }}$ is a regular background term, $v, \alpha, \beta$, and $\gamma$, are the usual critical exponents, and the $f_{i}(x)$ are various FSS functions with $x=\left(\beta-\beta_{c}\right) N^{1 / v D}$ being the scaling variable. The correction terms indicated by $[1+\cdots]$ become unimportant for sufficiently large system sizes $N$. We have expressed the scaling relations in terms of the total number of vertices $N$ rather than the linear size $L$ since the fractal dimension $D$ of the $\Phi^{3}$ random lattices is a priori unknown. Numerical simulations and various analytic approaches suggest that $D=4$ [49-52] for the ensemble of $\Phi^{3}$-gravity graphs we are considering, but we shall not need this explicitly for our analysis here. By rearranging the expression for $x$ one finally obtains the standard asymptotic scaling relation for the 
peak-locations (pseudo-critical points) $\beta_{\max , i}(N)$ on finite lattices,

$$
\beta_{\max , i}(N)=\beta_{c}+a_{i} N^{-1 / v D},
$$

with $a_{i}\left(=-x_{\max , i}\right)$ being a constant.

For the estimation of the critical exponents we can perform FSS fits of each of the quantities in (4) at all of the available pseudo-critical points. By monitoring the goodness-of-fit parameter $Q$ the fit range was adjusted to render fits of sufficiently high quality $(Q \geqslant 0.2$ is most cases). An overall estimate is then extracted by performing a direct or error weighted average. In both these cases we take a fairly conservative estimate for the errors by using the smallest contributing error bar. ${ }^{1}$

The dynamical aspects of the simulations are encoded in the autocorrelation functions and the associated integrated autocorrelation times $\hat{\tau}$. It is customary [44,45] to convert the $\hat{\tau}$ obtained in single-cluster simulations to a scale where, on the average, measurements are taken after every spin has been flipped once (similar to, e.g., Metropolis simulations), namely $\tau=f \hat{\tau}$ with $f=n_{\text {flip }}\langle|C|\rangle / N$. For quenched, random systems this procedure is not unique due to the necessary average over realizations, since one can take either $[\tau]_{\mathrm{av}} \equiv[f \cdot \hat{\tau}]_{\mathrm{av}}$ or $[f]_{\mathrm{av}} \cdot[\hat{\tau}]_{\mathrm{av}}$. The differences between the two averaging prescriptions turn out, however, to be extremely small in practice.

\section{Results}

\subsection{Planar $\Phi^{3}$ gravity graphs}

Let us start with the case of random $\Phi^{3}$ (pure) gravity graphs (without tadpoles or self-energy bubbles), where we studied the Potts models with $q=2$ and 4 on graphs of size $N=500,1000,2000,3000,4000,5000$, and 10000 [54,55], and the case $q=10$ with $N=250,500,1000,2000,3000,5000$, and 10000 [54,56,57], simulating in each case 64 realizations. It is well known that the pure $2 \mathrm{D}$ model exhibits second-order phase transitions for $q=2$ and 4 , but undergoes a rather strong first-order phase transition for $q=10$.

We present the results from the fitting and averaging procedure described above in Table 1. To give an indication of the quality of the data and fits we show in Fig. 3 the FSS of the susceptibility maxima for all three studied values of $q$. In obtaining the estimates in Table 1 we have dropped the smallest graph sizes and used the specific definition $U^{(1)}$ of the cumulants in all cases; the other two possibilities, e.g. $U_{4}^{(2)}=1-\left[\left\langle m^{4}\right\rangle\right]_{\mathrm{av}} / 3\left[\left\langle m^{2}\right\rangle^{2}\right]_{\mathrm{av}}$ and $U_{4}^{(3)}=1-\left[\left\langle m^{4}\right\rangle\right]_{\mathrm{av}} / 3\left[\left\langle m^{2}\right\rangle\right]_{\mathrm{av}}^{2}$, give values that are indistinguishable within the error bars. The results are quite stable to the deletion of the next smallest size, but the quality of the fits declines somewhat when this is done. The values in the last column were obtained from the hyperscaling relation

\footnotetext{
${ }^{1}$ The largest contributing error bar would certainly be too pessimistic; our choice probably errs on the side of caution too. In a more careful but much more elaborate analysis one would take into account the covariances of the different quantities as well [53].
} 
Table 1

$q$-state Potts model on $\phi^{3}$ random graphs: Fit results for the critical exponents

\begin{tabular}{lllllll}
\hline$q$ & Method & $1 / v D$ & $\gamma / v D$ & $\beta / v D$ & $(1-\beta) / v D$ & $\alpha / v D$ \\
\hline 2 & Weighted av. & $0.34(1)$ & $0.78(1)$ & $0.10(1)$ & $0.26(1)$ & $-0.32(1)$ \\
& Quenched & $0.3486 \ldots$ & $0.7094 \ldots$ & $0.1452 \ldots$ & $0.2033 \ldots$ & $-0.3027 \ldots$ \\
& KPZ & $0.3333 \ldots$ & $0.6666 \ldots$ & $0.1666 \ldots$ & $0.1666 \ldots$ & $-0.3333 \ldots$ \\
& Regular & 0.5 & 0.875 & 0.0625 & 0.4375 & 0 \\
4 & Weighted av. & $0.42(1)$ & $0.75(1)$ & $0.11(1)$ & $0.34(1)$ & $-0.16(1)$ \\
& Quenched & $0.5885 \ldots$ & $0.7094 \ldots$ & $0.1452 \ldots$ & $0.4433 \ldots$ & $0.1771 \ldots$ \\
& KPZ & 0.5 & 0.5 & 0.25 & 0.25 & 0.6875 \\
& Regular & 0.75 & 0.875 & 0.0625 & 0.5 & $0.16(1)$ \\
\hline
\end{tabular}

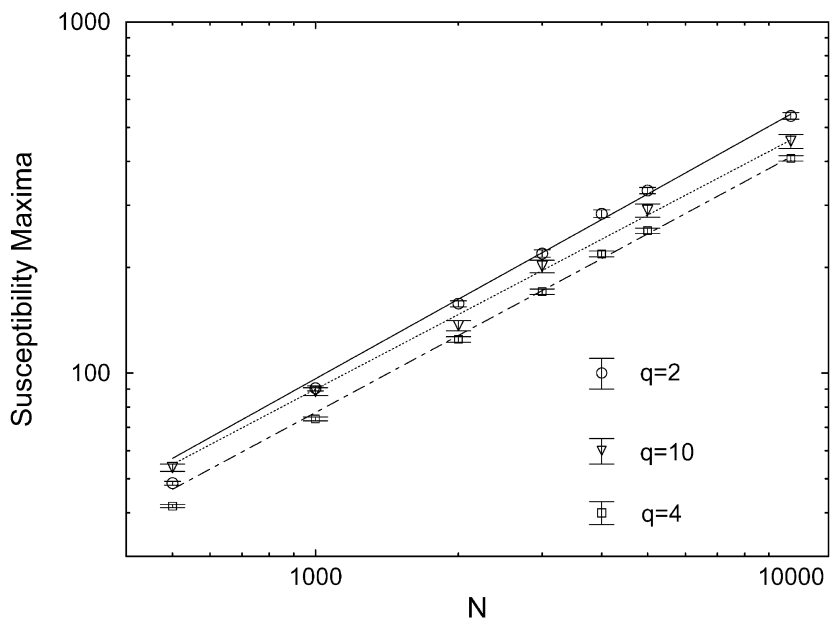

Fig. 3. Fits to $\chi_{\max }$ for $q=2,4$, and 10 .

$\alpha / v D=2 / v D-1$. Nonlinear three-parameter fits of the specific heat proved to be rather unstable for $q=2$ and 4 , and gave for $q=10$ only a rough estimate of $\alpha / v D=0.22(7)$. The FSS behaviour of $C$ is shown in Fig. 4. For comparison we have included in Table 1 the predictions of the quenched KPZ formula (2), the standard KPZ exponents and the regular $2 \mathrm{D}$ lattice exponents. Since the $q=10$ model has a first-order transition on a regular 2D lattice there are no direct predictions in this case.

Looking at the results in Table 1 it is clear that the exponent estimates are different from the exact values for regular 2D lattices, giving a clear indication that the connectivity disorder of planar random graphs is a relevant perturbation in the renormalization group sense, similar to the situation for random-bond disorder. Even more, for the 2D Ising model $(q=2)$ the values are unambiguously different while for random-bond disorder only rather subtle logarithmic modifications are expected which are difficult to observe in numerical studies [19-26]. Our estimates for $q=2$ are not incompatible with 


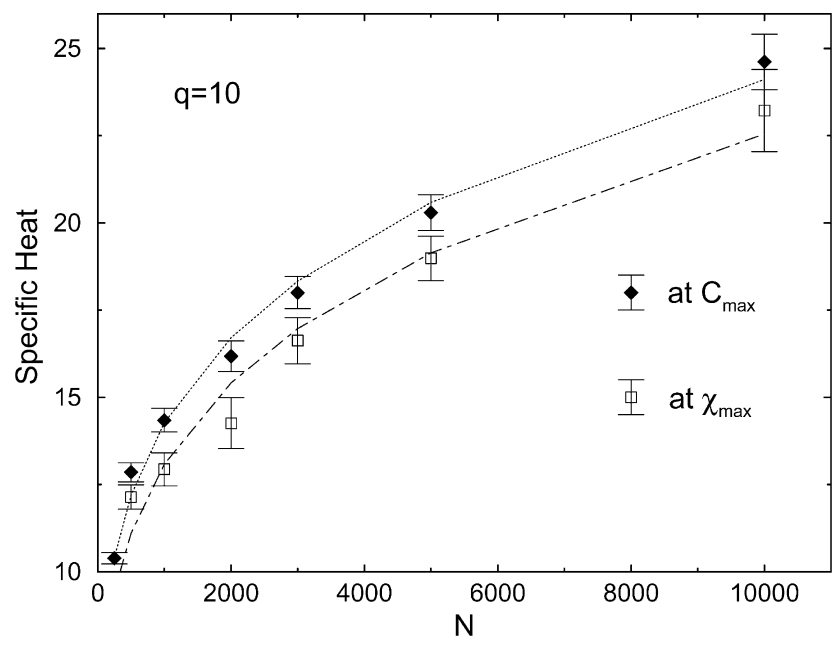

Fig. 4. Two representative fits (from the eleven used) for the FSS of the specific heat for $q=10$ evaluated at its own maximum and at the maximum of the susceptibility $\chi$.

both the quenched and KPZ values at the level of accuracy we have achieved, but that for $q=4$ definitely matches none of the possible theoretical predictions. Remarkably, the estimated $q=10$ values are a good fit to the theoretical quenched $q=4$ prediction; they are certainly incompatible with the $q=4 \mathrm{KPZ}$ values. It is also noteworthy that the $q=10$ measurements (and also the $q=4$ quenched theory predictions) violate a supposedly general bound $[58,59]$ for quenched systems, $1 / v D<1 / 2$. Hyperscaling implies that $\alpha / v D$ should be negative if the bound holds, which also is in clear conflict with our directly measured value for $q=10$. The numerical estimates of $1 / v D$ for $q=2$ and 4, on the other hand, are consistent with the bound. Whether the failure of the $q=10$ model to observe the bound is a consequence of the technical details of the averaging procedure as suggested in Ref. [60] or a result of long-range correlations in the disorder (which is due to the curvature correlations for the Liouville action in the original 2D gravity theory used to generate the graphs) is unclear.

In this context it is worth mentioning a closely related study [61] of the Ising model on quenched random graphs which formally can be characterized by a central charge $d=-5$. In this notation [43] our case corresponds to $d=0$. Even though the simulated $d=-5$ graphs were much smaller and the statistics poorer, in [61] very good agreement was obtained with the appropriate generalization of the quenched prediction (2).

Having determined $1 / v D$ we can extract the critical coupling $\beta_{c}$ from linear twoparameter fits to (5). Averaging all available estimates as before we obtained $\beta_{c}=$ $1.558(3), 1.835(1)$, and 2.244(1) for $q=2,4$, and 10, respectively. It is noteworthy that these values are very close to those found in the corresponding annealed simulations. We also tried to estimate $\beta_{c}$ from the crossing points of the fourth-order magnetic cumulants. The results for $\beta_{c}$ are consistent but less accurate, and for the cumulants at criticality we obtained the rough estimate $U_{4}^{*} \approx 0.55(3)$ for all three values of $q$. 
Let us finally briefly mention some results for autocorrelation times reported in detail in Ref. [62]. For $q=2$ our estimates of $\left[\tau_{e}\right]_{\mathrm{av}} \sim 3-4$ and $\left[\tau_{m}\right]_{\mathrm{av}} \sim 1.6-2.2$ are roughly independent of system size. For $q=4$ scaling behaviour is visible with $\left[\tau_{e}\right]_{\mathrm{av}} \sim 12-18$ and $\left[\tau_{m}\right]_{\mathrm{av}} \sim 7-10$, giving a dynamical exponent $z / D \sim 0.064(10)$ for the energy. Power-law scaling is much more pronounced for the $q=10$ model where $\left[\tau_{e}\right]_{\mathrm{av}} \sim 60-500$ and $\left[\tau_{m}\right]_{\mathrm{av}} \sim 40-350$ and much larger dynamical exponents $z / D \sim$ $\mathrm{O}(1)$ for both the energy and magnetisation have been obtained. More interesting are the self-averaging properties of the ensemble which can be investigated by considering the probability density for the $\tau$ 's, $P(\tau)$, rather than their average values. One would expect the cumulative distribution $F(\tau)=\int_{0}^{\tau} P\left(\tau^{\prime}\right) \mathrm{d} \tau^{\prime}$ to tend to a step function for increasing system size in a self-averaging system. This is observed not to be the case for all the models simulated, giving clear evidence of non-self-averaging behaviour. These observations can be put on a more quantitative basis by verifying [62] that $\Delta \tau /[\tau]_{\mathrm{av}}$ stays constant in the infinite-volume limit, where $\Delta \tau$ is the standard deviation. A nice graphical confirmation is the data collapse of $F(\tau)$ onto a single master-curve when plotted against the scaled variable $\tau /[\tau]_{\mathrm{av}}$.

\subsection{D Voronoi/Delaunay random lattices}

For the Ising $(q=2)$ model on 2D Voronoi/Delaunay lattices two independent MC studies as well as high-temperature series expansions gave no indications for a modified critical behaviour [63-66]. However, the 2D Ising model is a marginal case $\left(\alpha_{\text {pure }}=0\right)$ and disorder effects are difficult to observe also for random-bond models [19-25]. As bond disorder gave a clear signal for the 2D 8-state Potts model [6-9] it appeared promising to study the effect of connectivity disorder for the same model $[67,68]$. To this end we generated 20 independent realizations of 2D Voronoi/Delaunay random lattices with $N=250,500,750,1000,2000$, and 3000 sites and again performed long single-cluster simulations close to the transition point. Already an initial qualitative inspection of the data indicated, however, that the first-order nature of the phase transition in the pure model persists for this type of quenched connectivity disorder. The data of $C_{\max }$ and $\chi_{\max }$ shown in Fig. 5 is clearly consistent with a linear FSS behaviour, $C_{\max }=a_{C}+b_{C} N+\cdots$ and $\chi_{\max }=a_{\chi}+b_{\chi} N+\cdots$, characteristic for a first-order phase transition. Also shown are fits using this ansatz which yield $a_{C}=23.3(2.0), b_{C}=0.0659(30)$, with $Q=0.16$, and $a_{\chi}=-0.70(43), b_{\chi}=0.0629(13)$, with $Q=0.45$.

This observation is corroborated by the FSS of the pseudo-transition points, $\beta_{\max , i}=$ $\beta_{t}+a_{i} / N+\cdots$, which yield upon averaging over the estimates for $C, \chi$, and the energetic cumulants a final value of $\beta_{t}=0.83362(13)$. Further evidence for the persisting first-order nature of the transition can be obtained from the energy histograms which show a clear double-peak structure both for individual realizations and also after averaging. This also explains why here we could not afford to simulate bigger lattices: while cluster algorithms dramatically improve the performance of simulations at critical points, they cannot overcome the problem of exponential slowing down at first-order phase transitions which is caused by large free-energy barriers between 


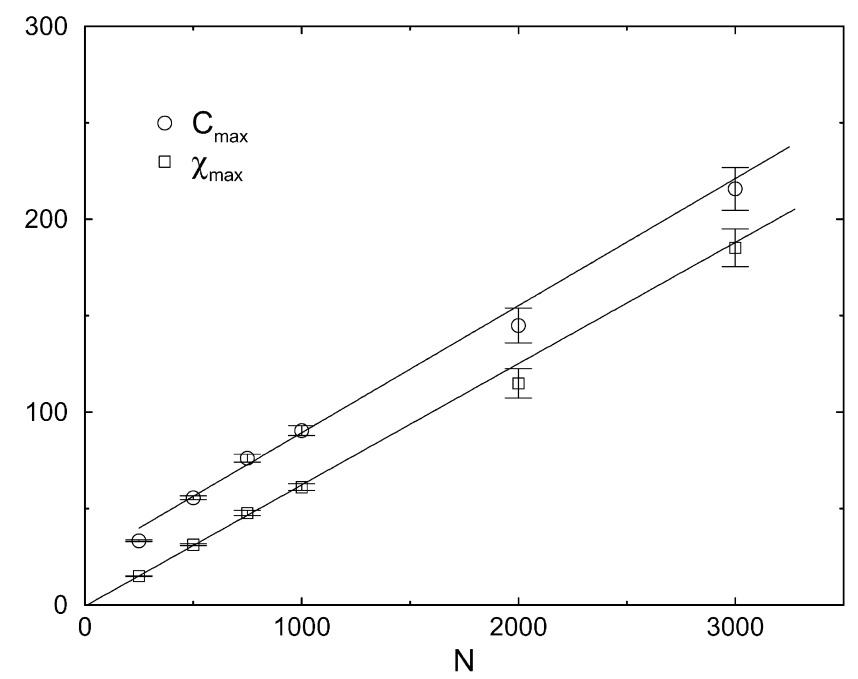

Fig. 5. FSS of specific-heat and susceptibility maxima of the 8-state Potts model on 2D Voronoi/Delaunay random lattices.

coexisting phases. In fact, refined methods particularly designed for the analysis of first-order phase transitions [69,70], which by their very definition rely on such signatures of coexisting phases, do confirm the conclusion derived by the more conventional FSS techniques. If in a future project one would wish to significantly enlarge the system sizes this would only be feasible with the help of multicanonical simulation techniques which, however, are quite cumbersome to implement for disordered systems (a successful implementation for spin glasses is reported in Refs. [71-74]).

\section{3. $3 D$ Voronoi/Delaunay random lattices}

In the $3 \mathrm{D}$ case the studied lattice sizes vary in powers of two from $N=2000 \approx 12.6^{3}$ to $N=128000 \approx 50.4^{3}$ [75]. For each size, we generated 96 randomly chosen lattice realizations. In $3 \mathrm{D}$ the probability density $P\left(q_{i}\right)$ is asymmetrically peaked around the mean value $\bar{q}_{i}=2+48 \pi^{2} / 35=15.5354 \ldots$, with tails extending down to $q_{i}=4$ and empirically up to about $q_{i}=36$ (for the actually studied lattice sizes and realizations).

In Ref. [75] we first concentrated on the Ising model for which it is well known that $\alpha_{\text {pure }} \approx 0.1>0$, so that according to the Harris criterion [2-5] one would a priori expect that quenched disorder is a relevant perturbation. MC simulations of the more standard case of site-dilution provided indeed evidence for new critical exponent values different from the pure model [79-81]. Similar conclusions can be drawn for 3D random-bond and bond-diluted Ising models from recently generated high-temperature series expansions [82]. As in the other cases the simulations were carried out with the single-cluster update algorithm close to the critical point. Starting from an initially completely ordered state about 30000 to 180000 clusters were discarded to reach equilibrium, and subsequently 100000 measurements of the energy and magnetisation were 
Table 2

Recent estimates of 3D Ising critical parameters $\quad(\mathrm{SCL}=$ simple cubic lattice, $\mathrm{VDRL}=\mathrm{Voronoi} /$ Delaunay random lattice)

\begin{tabular}{lllll}
\hline Method & $v$ & $\gamma / v$ & $\beta / v$ & $U_{4}^{*}$ \\
\hline SCL Ising [76] & $0.6289(8)$ & $1.970(14)$ & $0.518(7)$ & 0.47 \\
SCL Ising [77] & $0.6301(8)$ & $1.9630(30)$ & $0.5185(15)$ & $0.4652(4)$ \\
SCL $\phi^{4}$ [78] & $0.6296(3)(4)$ & $1.9642(4)(5)$ & - & $0.46555(9)$ \\
VDRL Ising [75] & $0.6299(5)$ & $1.9576(13)$ & $0.51587(82)$ & $0.4647(12)$ \\
\hline
\end{tabular}

recorded (with $n_{\text {flip }}$ adjusted to assure about $N / 2$ spin flips between measurements). For the integrated autocorrelation times we estimated $\left[\hat{\tau}_{e}\right]_{\mathrm{av}} \approx 2.5-3.5$ and $\left[\hat{\tau}_{m}\right]_{\mathrm{av}} \approx 2.4-3.0$ such that the statistics for each realization consists of about 15000-20000 effectively uncorrelated measurements, i.e., a total of $(1.5-2.0) \times 10^{6}$ almost uncorrelated data for each lattice size.

Performing the FSS analysis along the lines explained above we obtained from a total of 28 individual estimates of the correlation-length exponent a final weighted average of $1 / v=1.5875(12)$ or $v=0.6299(5)$, where we tacitly assumed that $D=3$. A comparison with recent numerical estimates for the standard $3 \mathrm{D}$ Ising universality class is compiled in Table 2. Once $v$ was known we could estimate the critical coupling from linear fits to be $\beta_{c}=0.0724249(40)$.

Repeating the fitting and averaging procedures for the other exponents as well we arrived at the values shown in Table 2. The comparison of our results with recent estimates for the pure 3D Ising model universality class clearly suggests that the connectivity disorder in Voronoi/Delaunay random lattices is not strong enough to modify the critical behaviour, contrary to the cases of random-bond disorder and random site-dilution. Of course, we cannot exclude the possibility of effective exponents which, for much larger lattice sizes, may exhibit a crossover to altered values governed by a different fixed point. In view of the very high quality of our fits to the leading FSS ansatz we can conclude, however, that very much larger lattices would be necessary to discover such a crossover.

\section{Conclusions}

Very high accuracy numerical simulations have shown that spin models on Poisonnian random lattices in both $2 \mathrm{D}$ and $3 \mathrm{D}$ stay stubbornly identical to their regular lattice brethren - there is no sign of the effects observed with quenched bond disorder [64-66]. However, a different picture emerges when one considers spin models living on a quenched ensemble of tri-valent $\left(\Phi^{3}\right)$ planar graphs, as generated by simulations of $2 \mathrm{D}$ quantum gravity. In this case the connectivity disorder is sufficiently strong for $q>4$ Potts model transitions to be softened to continuous transitions and for $q \leqslant 4$ exponents to be altered from their regular 2D lattice values. In this respect the connectivity disorder of such planar random graphs appears to be much more akin to 
random-bond disorder models than to Poisonnian random lattices. One very interesting feature of the $\Phi^{3}$ graphs is that exact, rather than perturbative, predictions for exponents exist in the $q \leqslant 4$ case, by virtue of taking a quenched limit in the KPZ formula.

The working hypothesis of the veracity of the quenched exponents is, however, at best only weakly supported by the numerical results. For the Ising $(q=2)$ model, the estimated value of $1 / v D$ is consistent with both the quenched and KPZ predictions. Although the estimates for the magnetic exponents $\gamma / v D, \beta / v D$, and $(1-\beta) / v D$ are closer to the quenched than the Onsager or KPZ values, any agreement is less than convincing. The estimated exponents of the $q=4$ model fit neither the quenched, KPZ nor regular lattice predictions, although one could argue that $\gamma / v D \sim 0.75(1)$ on its own is actually closer to the quenched prediction than the $q=2$ model. It is possible that $q=4$, which is subject to logarithmic corrections in both the regular lattice and KPZ cases, may require similar treatment in the quenched case but without more input on the form of these corrections fitting would be a futile exercise. The $q=10$ model, on the other hand, provides us with a set of estimated exponents that match the predicted quenched $q=4$ exponents extremely well but violate the bound $1 / v D<\frac{1}{2}$ of $[58,59]$, which merits an explanation in its own right.

Various other aspects of the behaviour of spin models on quenched random gravity graphs that have only been touched on here merit further investigation. The clear evidence of non-self-averaging behaviour for all $q$ and the autocorrelation scaling techniques used to quantify it are described in more detail in Ref. [62]. Similarly, the multifractal scaling of spin correlation functions merits also further investigation. Finally, as we have already noted, further simulations for other $q$ (and also $d$ ) values would help to determine whether the quenched exponents were correctly describing the behaviour of the models and cast further light on the remarkable, but possibly accidental, agreement between the $q=10$ simulations and the predicted $q=4$ exponents.

\section{Acknowledgements}

D.J. was partially supported by a Royal Society of Edinburgh/SOEID Support Research Fellowship. W.J. acknowledges partial support by the German-Israel-Foundation (GIF) under contract No. I-0438-145.07/95. The collaborative work of D.J. and W.J. was funded by ARC grant 313-ARC-XII-98/41. The numerical simulations were performed on a T3D parallel computer of Zuse-Zentrum für Informationswissenschaften Berlin (ZIB) under grant No. bvpf01.

\section{References}

[1] J. Cardy, Physica A 263 (1999) 215.

[2] A.B. Harris, J. Phys. C 7 (1974) 1671.

[3] Y. Imry, M. Wortis, Phys. Rev. B 19 (1979) 3581.

[4] M. Aizenman, J. Wehr, Phys. Rev. Lett. 62 (1989) 2503.

[5] K. Hui, A.N. Berker, Phys. Rev. Lett. 62 (1989) 2507; 63 (1989) 2433 (Erratum). 
[6] S. Chen, A.M. Ferrenberg, D.P. Landau, Phys. Rev. E 52 (1995) 1377.

[7] M. Kardar, A.L. Stella, G. Sartoni, B. Derrida, Phys. Rev. E 52 (1995) R1269.

[8] S. Wiseman, E. Domany, Phys. Rev. E 51 (1995) 3074.

[9] S. Wiseman, E. Domany, Phys. Rev. E 52 (1995) 3469.

[10] J. Cardy, J.L. Jacobsen, Phys. Rev. Lett. 79 (1997) 4063.

[11] J.L. Jacobsen, J.L. Cardy, Nucl. Phys. B 515 (1998) 701.

[12] C. Chatelain, B. Berche, Phys. Rev. Lett. 80 (1998) 1670.

[13] A.W.W. Ludwig, J.L. Cardy, Nucl. Phys. B 285 [FS19] (1987) 687.

[14] A.W.W. Ludwig, Nucl. Phys. B 285 (1987) 97.

[15] V. Dotsenko, V. Dotsenko, Sov. Phys. JETP Lett. 33 (1981) 37.

[16] V. Dotsenko, V. Dotsenko, Adv. Phys. 32 (1983) 129.

[17] V. Dotsenko, M. Picco, P. Pujol, Phys. Lett. B 347 (1995) 113.

[18] V. Dotsenko, M. Picco, P. Pujol, Nucl. Phys. B 455 (1995) 701.

[19] V.B. Andreichenko, Vl.S. Dotsenko, W. Selke, J.-S. Wang, Nucl. Phys. B 344 (1990) 531.

[20] J.-S. Wang, W. Selke, Vl.S. Dotsenko, V.B. Andreichenko, Europhys. Lett. 11 (1990) 301.

[21] J.-S. Wang, W. Selke, Vl.S. Dotsenko, V.B. Andreichenko, Physica A 164 (1990) 221.

[22] A.L. Talapov, L.N. Shchur, J. Phys: Condens. Matter 6 (1994) 8295.

[23] F.D.A. Aarão Reis, S.L.A. de Queiroz, R.R. dos Santos, Phys. Rev. B 56 (1997) 6013.

[24] D. Stauffer, F.D.A. Aarão Reis, S.L.A. de Queiroz, R.R. dos Santos, Int. J. Mod. Phys. C 8 (1997) 1209.

[25] A. Roder, J. Adler, W. Janke, Phys. Rev. Lett. 80 (1998) 4697.

[26] A. Roder, J. Adler, W. Janke, Physica A 265 (1999) 28.

[27] J. Cardy, J. Phys. A 32 (1999) L177.

[28] T. Olson, A.P. Young, Phys. Rev. B 60 (1999) 3428.

[29] C. Itzykson, Fields on random lattices, in: G.'t Hooft (Ed.), Progress in Gauge Field Theories, Proceedings Cargèse Summer School, Plenum Press, New York, 1983.

[30] M. Tanemura, T. Ogawa, N. Ogita, J. Comput. Phys. 51 (1983) 191.

[31] C.F. Baillie, D.A. Johnston, Mod. Phys. Lett. A 7 (1992) 1519.

[32] C.F. Baillie, D.A. Johnston, Phys. Lett. B 286 (1992) 44.

[33] J. Ambjørn, G. Thorleifsson, M. Wexler, Nucl. Phys. B 439 (1995) 187.

[34] J. Ambjørn, K.N. Anagnostopoulos, U. Magnea, G. Thorleifsson, Phys. Lett. B 388 (1996) 713.

[35] G. Thorleifsson, Nucl. Phys. B (Proc. Suppl.) 73 (1999) 133.

[36] V.G. Knizhnik, A.M. Polyakov, A.B. Zamolodchikov, Mod. Phys. Lett. A 3 (1988) 819.

[37] F. David, Mod. Phys. Lett. A 3 (1988) 1651.

[38] J. Distler, H. Kawai, Nucl. Phys. B 321 (1989) 509.

[39] W. Tutte, Can. J. Math. 14 (1962) 21.

[40] W. Tutte, Can. J. Math. 15 (1963) 249.

[41] M.E. Agishtein, A.A. Migdal, Nucl. Phys. B 350 (1991) 690.

[42] D.A. Johnston, Phys. Lett. B 277 (1992) 405.

[43] W. Janke, D.A. Johnston, Phys. Lett. B 460 (1999) 271.

[44] U. Wolff, Phys. Rev. Lett. 62 (1989) 361.

[45] U. Wolff, Nucl. Phys. B 334 (1990) 581.

[46] A.M. Ferrenberg, R.H. Swendsen, Phys. Rev. Lett. 61 (1988) 2635; 63 (1989) 1658 (Erratum).

[47] R.G. Miller, Biometrika 61 (1974) 1.

[48] B. Efron, The Jackknife, the Bootstrap and other Resampling Plans, SIAM, Philadelphia, PA, 1982.

[49] J. Ambjørn, K.N. Anagnostopoulos, T. Ichihara, L. Jensen, Y. Watabiki, JHEP 9811 (1998) 022.

[50] J. Ambjørn, K.N. Anagnostopoulos, G. Thorleifsson, Nucl. Phys. B (Proc. Suppl.) 63 (1998) 742.

[51] J. Ambjørn, K.N. Anagnostopoulos, Nucl. Phys. B 497 (1997) 445.

[52] M. Bowick, V. John, G. Thorleifsson, Phys. Lett. B 403 (1997) 197.

[53] W. Janke, M. Weigel, in preparation.

[54] W. Janke, D.A. Johnston, preprint hep-lat/9907026, July 1999, Nucl. Phys. B (in press).

[55] C.F. Baillie, K.A. Hawick, D.A. Johnston, Phys. Lett. B 328 (1994) 284.

[56] C. Baillie, W. Janke, D.A. Johnston, Phys. Lett. B 388 (1996) 14.

[57] C. Baillie, W. Janke, D.A. Johnston, Nucl. Phys. B (Proc. Suppl.) 53 (1997) 732.

[58] J.T. Chayes, L. Chayes, D.S. Fisher, T. Spencer, Phys. Rev. Lett. 57 (1986) 2999.

[59] J.T. Chayes, L. Chayes, D.S. Fisher, T. Spencer, Comm. Math. Phys. 120 (1989) 501. 
[60] F. Pázmándi, R.T. Scalettar, G.T. Zimányi, Phys. Rev. Lett. 79 (1997) 5130.

[61] K. Anagnostopoulos, P. Bialas, G. Thorleifsson, J. Stat. Phys. 94 (1999) 321.

[62] W. Janke, D.A. Johnston, preprint cond-mat/9911443, September 1999, J. Phys. A, in press.

[63] D. Espriu, M. Gross, P.E.L. Rakow, J.F. Wheater, Nucl. Phys. B 265 [FS15] (1986) 92.

[64] W. Janke, M. Katoot, R. Villanova, Phys. Lett. B 315 (1993) 412.

[65] W. Janke, M. Katoot, R. Villanova, Nucl. Phys. B (Proc. Suppl.) 34 (1994) 698.

[66] W. Janke, M. Katoot, R. Villanova, Phys. Rev. B 49 (1994) 9644.

[67] W. Janke, R. Villanova, Phys. Lett. A 209 (1995) 179.

[68] W. Janke, R. Villanova, Nucl. Phys. B (Proc. Suppl.) 47 (1996) 641.

[69] C. Borgs, W. Janke, Phys. Rev. Lett. 68 (1992) 1738.

[70] W. Janke, Phys. Rev. B 47 (1993) 14757.

[71] B.A. Berg, W. Janke, Phys. Rev. Lett. 80 (1998) 4771.

[72] W. Janke, B.A. Berg, A. Billoire, Ann. Phys. (Leipzig) 7 (1998) 544.

[73] W. Janke, B.A. Berg, A. Billoire, Comput. Phys. Commun. 121-122 (1999) 176.

[74] B.A. Berg, A. Billoire, W. Janke, Saclay preprint T99/095, September 1999, cond-mat/9910323, Phys. Rev. B, in press.

[75] W. Janke, R. Villanova, preprint, in preparation.

[76] A.M. Ferrenberg, D.P. Landau, Phys. Rev. B 44 (1991) 5081.

[77] H.W.J. Blöte, E. Luijten, J.R. Heringa, J. Phys. A 28 (1995) 6285.

[78] M. Hasenbusch, J. Phys. A 32 (1999) 4851.

[79] S. Wiseman, E. Domany, Phys. Rev. Lett. 81 (1998) 22.

[80] S. Wiseman, E. Domany, Phys. Rev. E 58 (1998) 2938.

[81] H.G. Ballesteros, L.A. Fernández, V. Martín-Mayor, A. Muñoz Sudupe, G. Parisi, J.J. Ruiz-Lorenzo, Phys. Rev. B 58 (1998) 2740.

[82] M. Hellmund, W. Janke, in preparation. 